\section{Motion Planning of a Climbing Parallel Robot}

\author{
M. Almonacid, R. J. Saltarén, R. Aracil, and O. Reinoso
}

\begin{abstract}
This paper proposes a novel application of the Stewart-Gough parallel platform as a climbing robot and its kinematics control to climb through long structures describing unknown spatial trajectories, such as palm trunks, tubes, etc. First, the description and design of the climbing parallel robot is presented. Second, the inverse and forward kinematics analysis of a mobile six-degrees-of-freedom parallel robot is described, based on spatial constraint formulation. Finally, the gait pattern and the climbing strategy of the parallel robot is described. The information from this research is being used in an actual climbing parallel robot design at Miguel Hernández University of Elche (Alicante), Spain.
\end{abstract}

Index Terms-Climbing robots, parallel robots, path planning, Stewart platform.

\section{INTRODUCTION}

Since proposed by Gough and Whitehall in 1962 and popularized by Stewart in 1965, the parallel platform has been used in many applications such as tire test machines [9], aircraft simulators [2], micro hands, and more recently, in large spherical radio telescopes. However, its application as a climbing robot has given impressive results [6]-[8]. The relatively small workspace and limited maneuverability of the Stewart platform are significantly improved when it is used as a mobile robot and some degrees of freedom (DOF) are aggregated. Also, the main features of this kind of robots are stiffness, high velocities, and high force-output-to manipulator-weight ratio, which makes them suitable for climbing applications, with very surprising results.

\section{A. Overview}

In this paper, we present the design of a parallel robot to climb through the capricious forms of the palm trunks and its kinematics control algorithm with on-line sensor-based correction for the path planning. The path-planning approach for 6-DOF parallel manipulators has been also addressed by other researchers [1], [3]. The paper is organized as follows. First, the design, kinematics modeling, and analysis of the parallel robot are presented. A numerical approach to solve the forward kinematics is chosen. In a following section, the gait pattern of the robot is described. The main feature of the climbing process consists of fixing one of the rings to the structure using a clamping system, and liberating, moving, and orienting the other ring to the planned point. Finally, the kinematics control algorithm of the robot is described. The purpose of the kinematics control algorithm is to provide the joint coordinates to the multiaxis control card, allowing the 6-DOF parallel robot

Manuscript received October 3, 2001; revised July 25, 2002. This paper was recommended for publication by Associate Editor M. Shoham and Editor I. Walker upon evaluation of the reviewers' comments. This work was supported by the Spanish Ministry of Education and Culture under Project 1FD1997-1338.

M. Almonacid is with the Systems and Automatic Engineering Department, Polytechnic University of Cartagena, 30202 Cartagena, Spain (e-mail: miguel.almonacid@upct.es).

R. J. Saltarén and R. Aracil are with the Systems, Electronic, Industrial Computing and Automatic Engineering Department, Polytechnic University of Madrid, 28006 Madrid, Spain (e-mail: rsaltaren@etsii.upm.es; r.aracil@etsii.upm.es).

O. Reinoso is with the Systems and Automatic Engineering Department, Miguel Hernández University of Elche, 03202 Elche-Alicante, Spain (e-mail: o.reinoso@umh.es).

Digital Object Identifier 10.1109/TRA.2003.810238

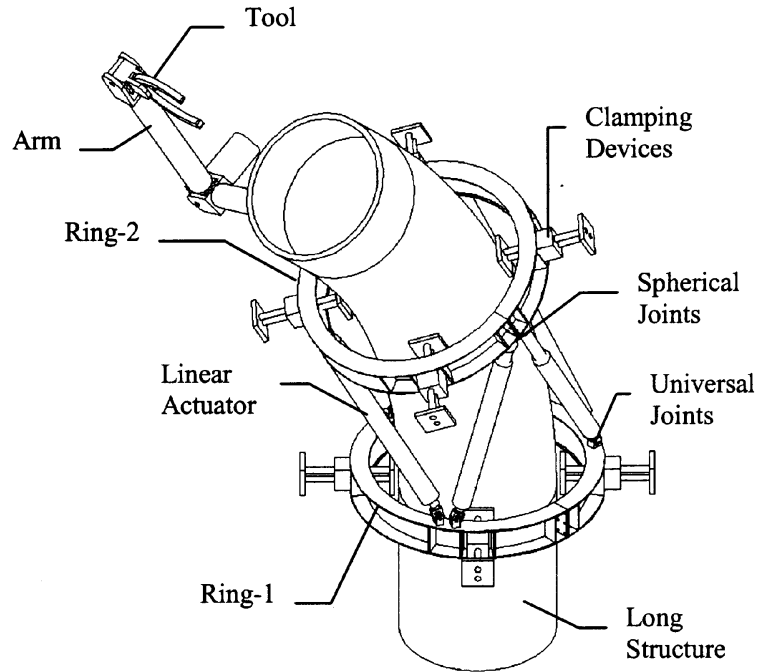

Fig. 1. Design of the climbing parallel robot (CPR).

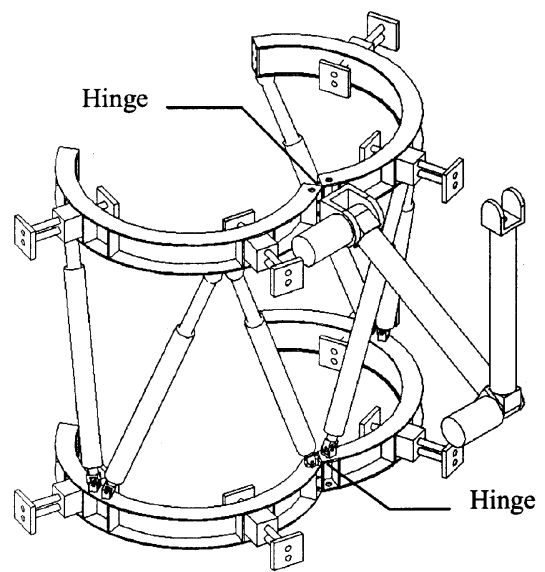

Fig. 2. Mechanical system aperture of the CPR.

to climb, autonomously, and pass through long structures describing unknown spatial trajectories.

\section{Description of the Climbing Parallel Robot (CPR)}

The six-universal-prismatic-spherical (UPS) parallel robot proposed as climbing robot, has a mechanical structure based on the Stewart platform with six DOFs. In Fig. 1, two rings are linked with six linear actuators. The actuators are linked to the base through universal joints, and to the upper platform through spherical joints. The actuators give to the robot six DOFs that are necessary for positioning and orienting itself in the workspace.

Taking into account the previous considerations, we have developed the design of a robot prototype that will be used to climb along curved tubular structures and turn around them in order to do different types of work, such as pruning and fumigation of palm trees, maintenance of oil pipes and electric posts, maintenance and inspection of steel cables, etc.

\section{A. CPR Working on Palm Trees}

To keep up the maintenance of palm trees, the CPR may be opened and assembled around the palm trunk. This problem is solved in Fig. 2. 


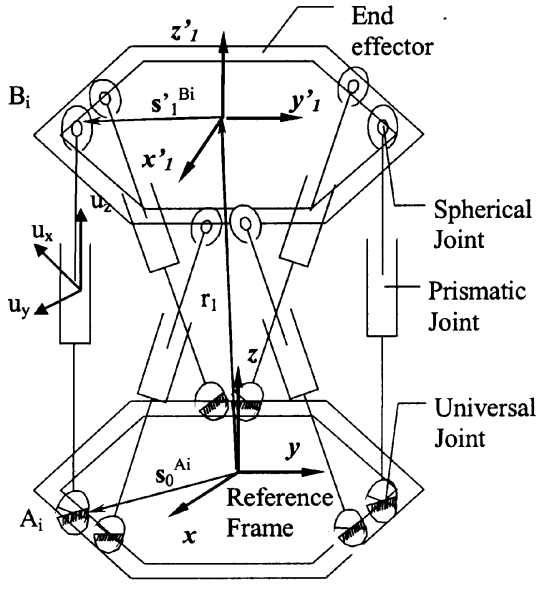

Fig. 3. Kinematic scheme of a 6-DOF parallel robot.

The CPR can be used in tasks such as fumigation, pollination, pruning, and harvest of high palm trees. These labors are of great importance for the treatment and maintenance of the Mediterranean palm groves. Nowadays, this type of task takes a lot of effort, due to the necessity of climbing to the top of the palm tree to perform them. To accomplish these tasks, the robot has to be able to take in its arm some specific tools (cutting, sprinkle, etc.). So, the human operator will be able to manage the robot in a teleoperated way, avoiding the risk of falling from palm trunks about $18 \mathrm{~m}$ high.

For the transportation of the robot between palm trees, we are considering a small mobile platform that makes easy the transport and assembly of the CPR around the palm tree trunk. This platform will include the power elements and the interface to teleoperate the climbing robot.

It is important to emphasize that in contrast with any other type of robot, a parallel robot is a mechanism very simple and easy to manufacture. Also, the autonomy of this type of robot is another very interesting factor to consider because of the low power consumption due to its light weight.

\section{KINEMATICS ANALYSIS}

In this section, the kinematics analysis of the parallel robot based on the spatial constraints formulation [4], [6] is carried out. First of all, the inverse kinematics problem is easily solved for robots with closed kinematics chains, and after that, we solved the forward kinematics problem, which turns out to be far more complex.

\section{A. Geometry of the CPR Prototype}

The configuration of the robot prototype consists of two regular hexagonal platforms and six identical linear actuators. We used hexagonal rings for the prototype of the robot, because in the faces of the ring we assemble the servovalves, control cards, and all the needed components for the control system. The legs are six UPS kinematics chains (where $\mathrm{U}$ is a universal joint connecting the base to the linear actuators, $\mathrm{P}$ is a prismatic DOF of the linear actuator, and $\mathrm{S}$ is the spherical joint connecting the linear actuator to the end-effector). The spatial parallel robot is shown in Fig. 3, where $x, y, z$ is the reference frame fixed at the base, and $x_{1}^{\prime}, y_{1}^{\prime}, z_{1}^{\prime}$ is the coordinate frame fixed at the center of gravity of the end-effector, with the $z_{1}^{\prime}$ axis pointing vertically upward.

\section{B. Inverse Kinematics}

The inverse kinematics allows the control of the relative motion between the rings of the robot. The inverse kinematics solution is calcu- lated using the position and orientation of the end-effector, described by the homogeneous matrix

$$
T_{1}=\left[\begin{array}{ccc|c}
n_{1} & s_{1} & a_{1} & p_{1} \\
\hline 0 & 0 & 0 & 1
\end{array}\right]=\left[\begin{array}{cc}
{\left[A_{1}\right]_{3 \times 3}} & {\left[r_{1}\right]_{3 \times 1}} \\
0 & 1
\end{array}\right]_{4 \times 4} .
$$

The inverse geometric model of a 6-UPS robot gives as a result the joint values of the kinematics chains, for a particular configuration of the end-effector. The solution can easily be obtained from the vector description on generalized coordinates

$$
\begin{aligned}
r_{A B i} & =r_{1}+A_{1} s_{1}^{\prime B i}-s_{0}^{A i}, \quad i=1, \ldots, 6 \\
C_{i} & =\left\|r_{A B i}\right\|
\end{aligned}
$$

where $s_{0}^{A i}$ and $s^{\prime B i}$ are the position vectors that locate the universal and spherical joints $A_{i}$ and $B_{i}$, with respect to the base reference frame and the mobile ring coordinate system, respectively. The $r_{A B i}$ vectors are the joint variables calculated from the inverse solution, whose magnitude $C_{i}$ gives the required configuration of the linear actuators.

\section{Forward Kinematics}

The forward kinematics allows obtaining the new configuration of the robot, once one of its rings has been fully extended or gathered.

In general, a 6-DOF UPS parallel robot consists of 14 bodies (parts), formed by the couplings of the six linear actuators (each actuator is composed by two parts), the end-effector (ring 2), and the reference base (ring 1). Taking ring 1 as a ground reference, the rest of the parts of the robot $(n b=13)$ can be modeled through the generalized coordinates vector $q=\left[q_{1}, q_{2}, q_{i}, \ldots, q_{n b}\right]^{T}$. Each component is a generalized coordinates vector $q_{k}=\left[x_{k}, y_{k}, z_{k}, e_{0}^{k}, e_{1}^{k}, e_{2}^{k}, e_{3}^{k}\right]_{7 \times 1}^{T}$ described as a function of the Euler parameters.

The kinematics chain of the robot is modeled through the constraint equations $\phi(q)=0$. These constraint equations define in a unique way all the mechanical joints (universal, spherical, and prismatic) among the $n b$ parts of the robot kinematics chain. Furthermore, it includes the kinematics restrictions imposed by the actuators' DOFs.

In practice, we have used a reduced generalized-coordinates vector, discarding artificially one of the two parts of each actuator, and making the prismatic and universal joints coincide on the same point. Using the previous simplification, the number of parts to model is reduced to seven $(n b=7)$. This assumption allows us to have the next reducedconstraint equations

$$
\phi(q)=\left[\begin{array}{c}
\phi^{K}(q) \\
\phi^{D}\left(q, C_{i}\right) \\
\phi^{P}(q)
\end{array}\right]_{49 \times 1}=0
$$

where $\phi^{K}(q)_{36 \times 1}=0$ is a vector of holonomic constraints imposed by prismatic, spherical, and universal joints. $\phi^{D}\left(q, C_{i}\right)_{6 \times 1}=0$ is a vector of six kinematic constraints that describe the displacement $C_{i}$ of each actuator. $\phi^{P}(q)_{7 \times 1}=0$ is a vector of seven constraints for the normalization of Euler parameters.

To solve the forward kinematics model based on the constraint equations (4), we have used the Newton-Raphson method.

\section{Path Planning}

The climbing procedure consists of fixing one of the rings (the inertial reference frame) to the structure using a clamping system, as long as the other ring-clamping system can be loose in order to move and orient to the planned point. For path planning, a coordinate frame $x_{1}^{\prime}, y_{1}^{\prime}, z_{1}^{\prime}$ is fixed at the center of mass of the moving platform.

\section{A. Perception System}

In order to make the CPR to follow any unknown trajectory, it needs some environmental information. Thus, three ultrasonic sensors placed 


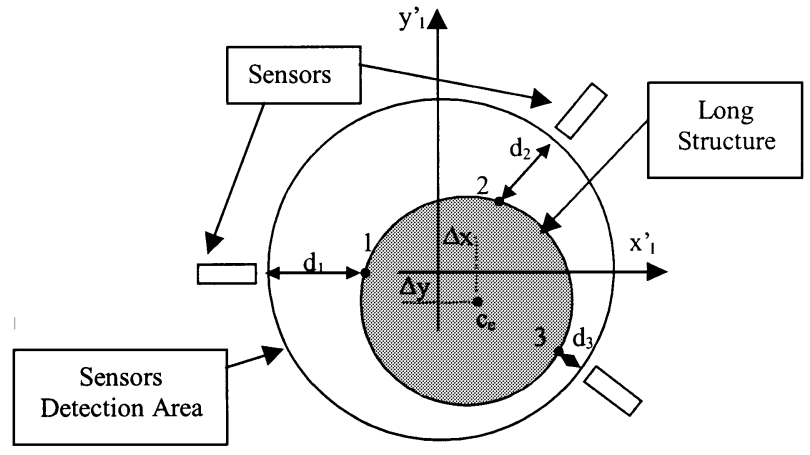

Fig. 4. Correction function.

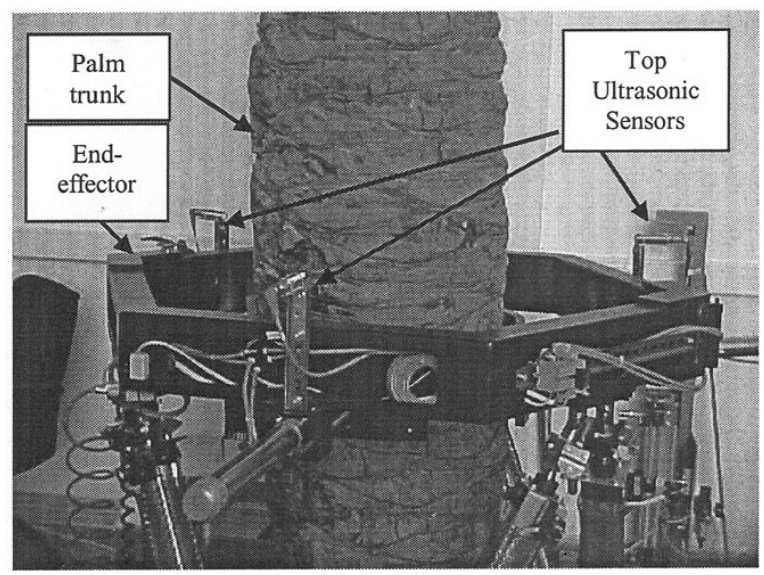

Fig. 5. End-effector of the CPR.

on each ring and separated $120^{\circ}$ provide this information, see Fig. 4. A picture of the prototype with the allocation of the top ultrasonic sensors at the end-effector is shown in Fig. 5.

\section{B. Correction Function}

The allocations of the ultrasonic sensors define a plane where the measures are taken. The sensorial information allows knowing the position of the center of the structure on the plane of the platform. The intersection of this plane with the long structure allows us to calculate the corrections needed for the centering of the platform, Fig. 4. The distances measured by the ultrasonic sensors $\left(d_{1}, d_{2}, d_{3}\right)$ give the information to determine the deviation $(\Delta x, \Delta y)$ of the center of the structure $\left(c_{e}\right)$, with respect to the coordinate frame of the platform $\left(x_{1}^{\prime}, y_{1}^{\prime}\right)$.

The allocation of the sensors is known. The distances $d_{i}(i=1,2,3)$ give the coordinates of the points 1,2 , and 3 . These points define a circle whose center matches with the structure one. Therefore, to determine the deviation $(\Delta x, \Delta y)$, it is necessary to solve the next system of equations

$$
R=\sqrt{\left(x_{i}-\Delta x\right)^{2}+\left(y_{i}-\Delta y\right)^{2}}
$$

where $\left(x_{i}, y_{i}\right)$ for $i=1,2,3$ are the coordinates of the points and $R$ is the radius of the structure circle.

\section{Input Parameters}

The inputs of the control algorithm are:

- the number of cycles: $n_{\text {cycles }}$;

— the quantity of displacement: $\Delta$.

A cycle corresponds to a complete advancement and retrieval of the linear actuators. The path planning of the moving platform is discretized by the input $\Delta$ quantity in each semicycle. This quantity is

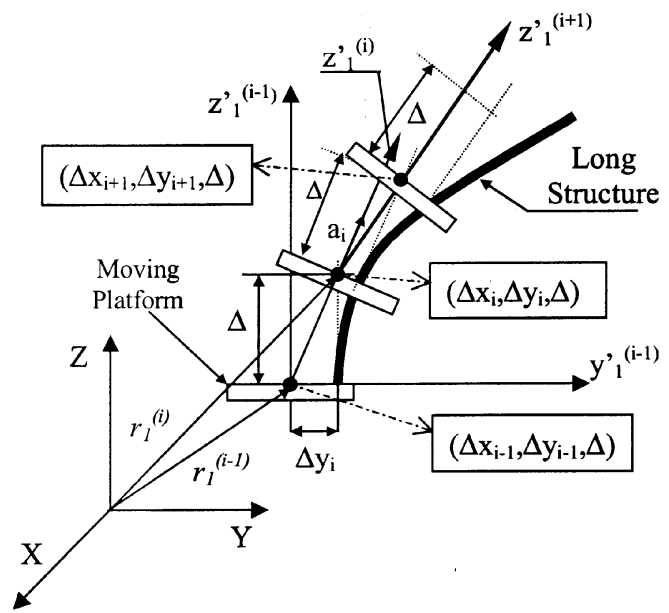

Fig. 6. Prediction function.

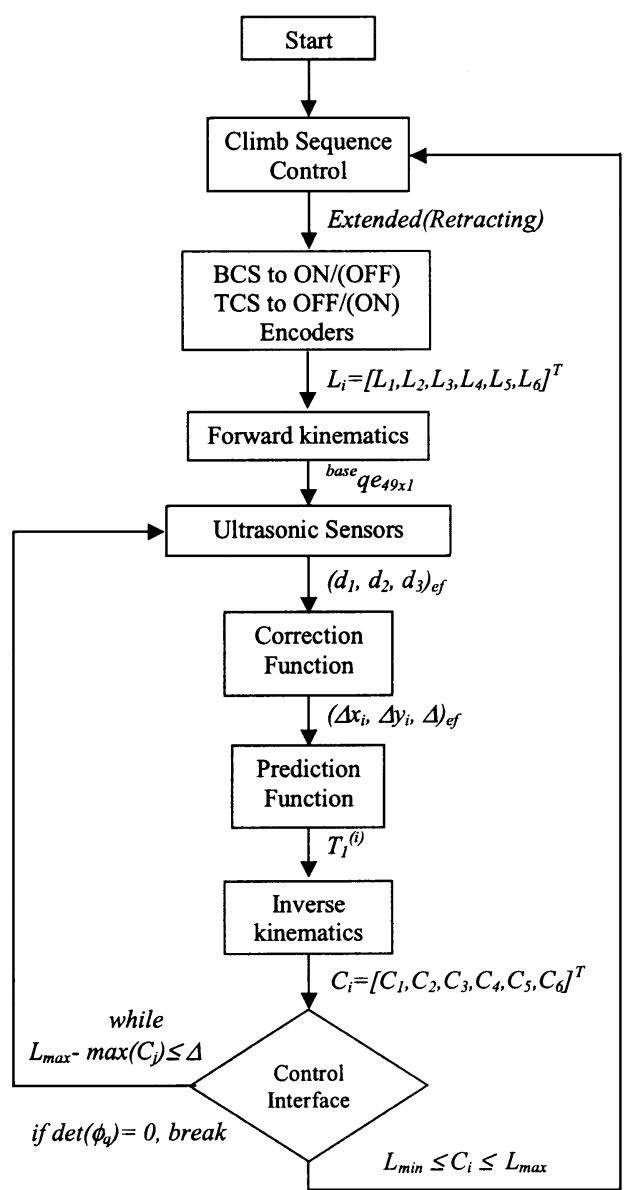

Fig. 7. Kinematics control algorithm.

fixed depending on the curvature of the structure. It is also possible for this quantity not to be fixed. It can be a function of the curvature ratio of the structure during the climbing process, and even to vary as a function of this ratio.

\section{Prediction Function}

The aim of the prediction function is to calculate the new position and orientation of the moving platform at each step (Fig. 6).

First, the new planned position $r_{1}^{(i)}=[x, y, z]^{T}$ of the moving platform is calculated. The platform is displaced a prefixed discretized 


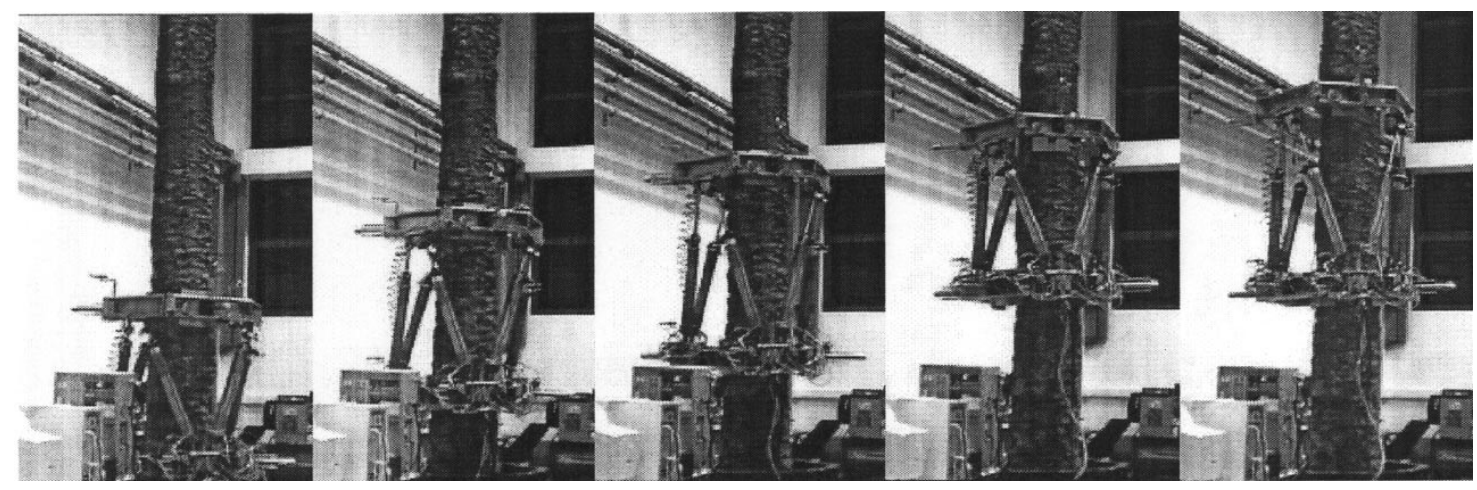

Fig. 8. Experimental results of the application of the kinematics control algorithm to the CPR prototype.

quantity $(\Delta)$ along the $z^{\prime(i-1)}$ axis, and at the same time, it is corrected with the deviation $\left(\Delta x_{i}, \Delta y_{i}\right)$ previously calculated in the platform plane by the corrector function. In this sense, the location of the new coordinate frame $x_{1}^{\prime(i)}-y_{1}^{\prime(i)}-z^{\prime(i)}$ corresponds to the position $\left(\Delta x_{i}, \Delta y_{i}, \Delta\right)$ with respect to the $x_{1}^{\prime(i-1)}-y_{1}^{\prime(i-1)}-z_{1}^{\prime(i-1)}$ coordinate frame attached to the moving platform, as is shown in Fig. 6.

Given the position $r_{1}^{(i-1)}$ and the rotation matrix $A_{1}^{(i-1)}$ of the moving platform at time $t$, the new planned position of the moving platform with respect to the reference frame $X-Y-Z$ is given by

$$
r_{1}^{(i)}=r_{1}^{(i-1)}+A_{1}^{(i-1)} \cdot\left[\Delta x_{i}, \Delta y_{i}, \Delta\right]^{T} .
$$

The new planned orientation of the moving platform is obtained with the determination of the unit vectors along the $x_{1}^{\prime(i)}, y_{1}^{\prime(i)}$, and $z_{1}^{\prime(i)}$ axes, commonly labeled as $n$ (normal), $s$ (sliding), and $a$ (approach). A prediction for the approach direction of the platform can be obtained by a vector joining the positions of both the previous and new coordinate frames. The new $z_{1}^{\prime(i)}$-axis direction of the moving platform will be aligned with this approach vector $a_{i}$. The corresponding $x_{1}^{\prime(i)}$-axis and $y_{1}^{\prime(i)}$-axis unit vectors $n_{i}$ and $s_{i}$, respectively, are calculated by the cross product in relation to the previous orientation vector $y_{1}^{(i-1)}$ as

$$
\begin{aligned}
a_{i}= & {\left[r_{1}^{(i)}-r_{1}^{(i-1)}\right] /\left\|r_{1}^{(i)}-r_{1}^{(i-1)}\right\| } \\
n_{i}= & {\left[y_{1}^{(i-1)} \times a_{i}\right] /\left\|y_{1}^{(i-1)} \times a_{i}\right\| } \\
& s_{i}=a_{i} \times n_{i} .
\end{aligned}
$$

The unit vectors in (7) form the new rotation matrix of the moving platform $A_{1}^{(i)}$ as

$$
A_{1}^{(i)}=\left[n_{i}, s_{i}, a_{i}\right] .
$$

\section{E. Kinematics Control Algorithm}

The purpose of the kinematics control algorithm is to provide an on-line sensor-based guidance strategy to the 6-DOF CPR in order to climb autonomously and through unknown long structures.

The kinematics control algorithm is referred to as predictive because the error is detected in one step and it is corrected in the next one as the robot moves along the structure.

1) Block Diagram: The block diagram of the kinematics control algorithm is shown in Fig. 7. The algorithm consists of the following steps.

1) The bottom clamping system (BCS) at the lower ring (ring 1) is activated, setting the reference system at the base of the robot. The top clamping system (TCS) gets off. In this way, the upper ring (ring 2) is free to move. The actuator's length, measured by the encoders $L_{i}$ and the forward kinematics, gives the configuration of the robot in a vector ${ }^{\text {base }} q e_{49 \times 1}$ of generalized coordinates.
2) From distances of the top ultrasonic sensors $\left(d_{1}, d_{2}, d_{3}\right)_{e f}$, the correction function calculates the deviation $\left(\Delta x_{i}, \Delta y_{i}\right)$ of the center of the long structure with respect to the center of the ring.

$3)$ Given the specified movement quantity $(\Delta)$ and the calculated deviation $\left(\Delta x_{i}, \Delta y_{i}\right)$, the prediction function (6)-(8) calculates the new planned position and orientation of the end-effector $T_{1}^{(i)}$ (ring 2). In each cycle after step 1, we use the relation between Euler parameters and the associated transformation matrices $A=\left(2 e_{0}^{2}-1\right) I+2\left(e e^{T}+e_{0} \tilde{e}\right)$, [4].

4) The inverse kinematics (2)-(3) calculate the corresponding joint coordinates in a vector $C_{i}=\left[C_{1}, C_{2}, \ldots, C_{6}\right]^{T}$.

5) The displacement vector $C_{i}$ calculated in the previous step is sent to a path planner to generate the command vector to each actuator. The process will continue to step 4 until the control interface indicates the distance limit, that is, the advancement in one of the cylinders plus the selected movement $(\Delta)$ exceeds the maximum distance covered by the actuator $\left(L_{\max }-\max \left(C_{i j}\right) \leq \Delta\right.$ ), or a singular configuration is detected by the Jacobian matrix $\left(\phi_{q}\right)$ determinant [1], [6].

6) Return to step 1 , but the climbing sequence control activates the TCS at ring 2, clamping this ring around the palm trunk, and the BCS of ring 1 gets off. In this way, ring 1 is free to climb up.

Steps 1-6 are repeated until the robot arrives at the top of the palm tree.

Through inverse and direct kinematics and clamping each ring in an alternative sequence, the robot is commanded to turn around the palm trunk, and so the arm can be placed in any position.

The descend of the robot is possible repeating in reverse order steps $1-6$ of this algorithm.

\section{CONCLUSIONS}

The suitability of the Stewart platform used as a climbing robot has been pointed out. A control algorithm for the path planning of the parallel robot has been developed. The gait pattern is shown and a kinematics control algorithm for climbing through unknown spatial trajectories has been implemented. The kinematics analysis of the robot based on the spatial formulation of constraints has been carried out. The results of this research are being applied in an actual CPR design at Miguel Hernandez University, Elche, Spain, Fig. 8.

The development of advanced technological means, for the maintenance of palms, has been outlined as high priority for the population of palms of the city of Elche, in the county of Alicante, Spain. The objective is to try to preserve a historical patrimony of more than 2000 years and with more than 180000 specimens, recently declared a Patrimony of Humanity by UNESCO, and that is in clearing deterioration from the attack of plagues and manpower lack for its maintenance, due to 
the high risk factor implied in going up to heights between 12 and 18 m.

\section{REFERENCES}

[1] B. Dasgupta and T. S. Mruthyunjaya, "Singularity-free planning for the Stewart platform manipulator," Mech. Mach. Theory, vol. 33, no. 6, pp. $711-725,1998$

[2] D. Stewart, "A platform with six degrees of freedom," Proc. Instr. Mech. Engs., vol. 180-1, no. 15, pp. 371-386, 1965.

[3] D. N. Nenchev and M. Uchiyama, "Singularity-consistent path planning and control of parallel robot motion through instantaneous self-motion type," in Proc. IEEE Int. Conf. Robotics and Automation, Minneapolis, MN, Apr. 24-26, 1996, pp. 1864-1870.

[4] E. J. Haug, Computer-Aided Kinematics and Dynamics of Mechanical Systems. Boston, MA: Allyn and Bacon, 1989.

[5] J. P. Merlet, Les Robots Parallèles. Liege, Belgium: Ed. Hermes, 1997.

[6] M. Almonacid, S. K. Agrawal, R. Aracil, and R. J. Saltaren, "Multibody dynamic analysis of a 6-DOF parallel robot," in Proc. ASME Int. Symp. Advances in Robot Dynamics and Control, New York, NY, 2001.

[7] R. Aracil, R. Saltarén, J. M. Azorin, M. Almonacid, and J. M. Sabater, "Climbing parallel robots morphologies," in Proc. 6th Int. IFAC Symp. Robot Control, vol. 1, Austria, Sept. 2000, pp. 145-150.

[8] R. Saltarén, R. Aracil, O. Reinoso, J. M. Sabater, and M. Almonacid, "Parallel climbing robot for construction, inspection and maintenance," in Proc. Int. Symp. Automation and Robotics in Construction, Madrid, Spain, 1999, pp. 359-364.

[9] V. E. Gough and S. G. Whitehall, "Universal tyre test machine," in Proc. 9th Int. Technology Congr. FISITA, May 1962, pp. 117-137.

\section{Vibration Control of Gough-Stewart Platform on Flexible Suspension}

Yuan Cheng, Gexue Ren, and ShiLiang Dai

\begin{abstract}
This paper studies the vibration control of the Gough-Stewart platform on flexible supporting structures. The distinct characteristic of this application is that the two platforms are dynamically coupled. A proportional derivative control law based on the position prediction is used in the system. The control effects are evaluated by comparing the root mean square responses of the two platforms.
\end{abstract}

Index Terms-Flexible supporting structure, Gough-Stewart platform, vibration control.

\section{INTRODUCTION}

The Gough-Stewart platform [1] has the capability to control the six degrees of freedom (DOFs) of its moveable platform, and has been proven to be of high positioning accuracy while maintaining a high force-to-weight ratio. So, it is chosen to stabilize the receivers of a

Manuscript received July 25, 2002; revised November 13, 2002 and December 11, 2002. This paper was recommended for publication by Associate Editor H. Arai and Editor I. Walker upon evaluation of the reviewers' comments. This work was supported by the Research Fund for Large Radio Telescopes, Chinese National Astronomical Observatories; the Fundamental Research Fund of Tsinghua University under No. JC1999031; and the Natural Science Foundation of China under Project 10172049.

The authors are with the Department of Engineering Mechanics, Tsinghua University, Beijing100084, China (e-mail: ycheng99@mail.tsinghua.edu.cn; Rengx@mail.tsinghua.edu.cn).

Digital Object Identifier 10.1109/TRA.2003.810233

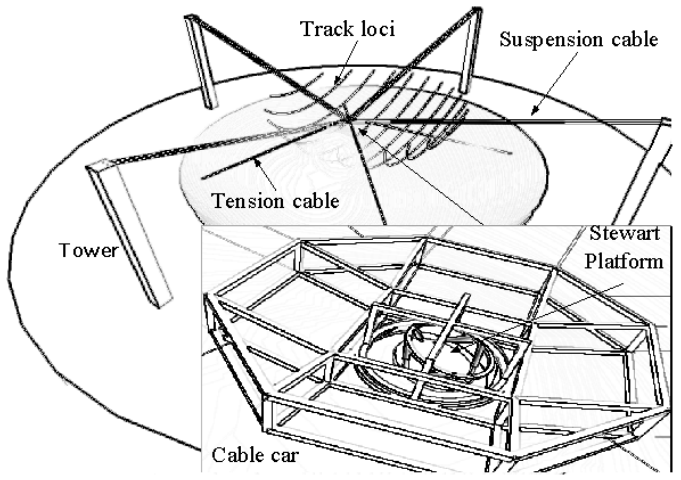

Fig. 1. Suspending structure of the radio telescope.

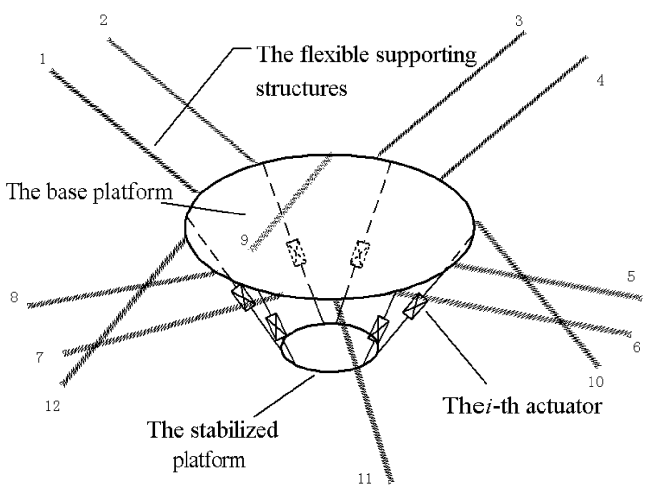

Fig. 2. Working diagram of the elastically restrained Stewart platform. 8: suspension cables. 9-12: pretension stabilized cables.

500-m aperture radio telescope [2], which has a spherical focal surface with an aperture of $250 \mathrm{~m}$, and the focal surface is $150 \mathrm{~m}$ above the reflector (see Fig. 1). To observe 5-GHz radio waves, the astronomical receivers are required to follow tracks on the focal surface with positioning precision of $4 \mathrm{~mm}$ in position and $0.1^{\circ}$ in orientation. In the platformless feed-support concepts [3], [4], the receivers are either driven by cables or carried by a cable car. The expected wind-induced vibration might be as high as $0.5 \mathrm{~m}$ in position and $3^{\circ}$ in orientation, much higher than the specifications. The Gough-Stewart platform in this application is shown in Fig. 2. The flexible supporting structure (including eight suspension load cables and four pretension stabilized cables) supports the base platform (cable car). The arm is to isolate the vibration by adjusting the six actuators with appropriate control laws when the cable car vibrates with wind load.

There are many publications on the dynamics and control of the Gough-Stewart platform [5]-[11], but in most of these works, the base platform is fixed on the ground. Studies [12]-[15] have investigated the use of the Gough-Stewart platform for vibration isolation, but they are concentrated on vibrations of relatively small amplitude, and as the inertia of the base is much larger than that of the payload, the coupling effect of the two platforms is not prominent.

In the application presented in this paper, as the base platform is not fixed on the ground, the reaction force caused by the motion of the stabilized platform will lead to perturbation on the base platform, and will induce vibration of the whole system. In this paper, a simple control law of the prediction of the base platform and proportional derivative (PD) feedback is used to study the feasibility of the system. The control effects are simulated by the multibody method. 